\title{
Osteochondral Tissue Engineering Constructs with a Cartilage Part Made of Poly(L-Lactic Acid) / Starch Blend and a Bioactive Poly(L-Lactic Acid) Composite Layer for Subchondral Bone
}

\author{
S. Ghosh ${ }^{1,3}$, J.C. Viana ${ }^{2,3}$, R.L. Reis ${ }^{1,3}$, J.F. Mano ${ }^{1,3}$ \\ ${ }^{1} 3 \mathrm{~B}$ 's Research Group - Biomaterials, Biodegradables and Biomimetics, Campus de \\ Gualtar, 4710-057 Braga, Portugal. \\ ${ }^{2}$ IPC- Institute for Polymers and Composites, \\ ${ }^{3}$ Department of Polymer Engineering, University of Minho, 4800-058, Campus De Azurem, \\ Guimarães, Portugal \\ jmano@dep.uminho.pt
}

Keywords: Osteochondral scaffolds, Tissue engineering, Articular cartilage, Biodegradable polymers, Bioactivity

\begin{abstract}
Articular cartilage has an inadequate natural rebuilding capacity. Tissue engineering has shown to have potential to provide an effective alternative to engineer the damaged cartilage. In this study, an integrated porous bi-layered scaffold was developed aiming to mimic the requirements of cartilage and underlying subchondral bone. The osteochondral approach explored in this work was to include a common polymeric component in both cartilage and bone components, which maximised the integration at the interface by mean of a melt-based processing route. A blend of starch and poly(Llactic acid),PLLA, was used in the cartilage side, which was found to possess an adequate water uptake capability. For the bone region, to induce bioactivity, PLLA had been reinforced with hydroxyapatite (HA) and bioactive glass (BG). The surfaces of the constructs were investigated as a function of soaking time in a simulated body (SBF) fluid using scanning electron microscopy (SEM) and FTIR. The SEM - FTIR indicated a bone-like apatite formation and the surface coverage by apatite layer increased with increasing soaking time, whereas the cartilage-layer did not exhibit the formation of any apatite like layer.
\end{abstract}

\section{Introduction}

The repair of articular cartilage, as a result of trauma, tumour resection or degeneration, remains an intractable problem, due to poor natural healing capacity of this tissue owing to its avascular nature [1]. Current clinical approaches to enhance the natural rebuilding of articular surfaces include modifications of the damaged surface (chondral shaving with debridement, abrasion arthroplasty, subchondral drilling or microfracturing of the subchondral bone) and transplantation of periosteal, perichondral [2,3] or osteochondral [4] autografts. However, none of these currently available therapies can provide a long-term solution to refurbish an enduring cartilage healing [5].

Tissue engineering approaches have a great potential to lead to the development of strategies for the biological and functional regeneration of cartilage and of osteochondral defects. An important factor in the fabrication of a porous bi-layered construct is the need of a good integration between the two compartments, avoiding the construct delamination and promoting the long-term integrity of the articular cartilage surface.

For the subchondral bone, poly(L-lactic acid), PLLA, may be an adequate choice as matrix material because of superior mechanical properties and biodegradability [6]. The use of ceramics, namely hydroxyapatite and bioglass, along with PLLA showed good osteoconductivity and some degree of biocompatibility both in-vivo and in-vitro $[7,8]$.

It was also suggested that PLLA is also a suitable substrate for scaffolding materials for cartilage tissue-engineering [9]. In this context, it may be advantageous to associate PLLA with naturalbased materials, as they usually show good interaction with cells and improve the hydrophilic 
character of the polymer. In this work, the cartilage component of the developed constructs was produced with a blend of PLLA and corn starch. It was shown that such blends could exhibit less cytotoxicity than pure PLLA, and a comparable adhesion and proliferation of osteoblasts-like cells [10]. The bi-layered construct is processed using an organic solvent-free methodology, based on compression moulding and salt leaching, and the bioactive character of both layers is investigated.

\section{Materials and methods}

\subsection{Materials}

The PLLA, used in this work was of high stereoregularity and had $M_{n}=69,000$ and polydispersity of 1.73. The blend of PLLA and starch used in this study contained $50 \mathrm{wt} \%$ of starch and was labelled SPLA50.

The hydroxyapatite (HA) used in this study was supplied by Plasma Biotal Ltd, U.K. This sintered HA had an average particle size of $10 \mu \mathrm{m}$. The Bioglass 45S5 (BG) used in this study was from Novamin Technology Inc., Florida, USA. The particle sizes was ranging from 3.8 to $5.3 \mu \mathrm{m}$

\subsection{Fabrication of the scaffolds}

PLLA and SPLA50 were pre-dried at $50{ }^{\circ} \mathrm{C}$ at $100 \mathrm{mbar}$ for 4 hours in a vacuum oven. The polymers were cryogenically milled with liquid nitrogen and sieved by a strainer of $500 \mu \mathrm{m}$ mesh size. $\mathrm{NaCl}$ particles of 250 to $500 \mu \mathrm{m}$ and $<125 \mu \mathrm{m}$ particle size were sieved.

For the bone side, a 70/30 wt $\%$ PLLA/HA or PLLA/BG mixture was blended with $70 \mathrm{wt} \% \mathrm{of} \mathrm{NaCl}$ (particle size $=250-500 \mu \mathrm{m}$ ). For the cartilage side, SPLA50 was blended with $80 \mathrm{wt} \%$ of $\mathrm{NaCl}$ (particle size $<125 \mu \mathrm{m}$ ). The bi-layered construct was 1:1 wt\% proportion of the two components.

The blends were dried at $50{ }^{\circ} \mathrm{C}$ at 40 mbar for 4 hours in a vacuum oven prior to compression moulding. To produce the bi-layered construct, the PLLA-HA-NaCl or PLLA-BG-NaCl mixtures were first put into the compression moulding machine, compressed by 5 tons load for 30 seconds and after this process the load was removed. The SPPLA50+ $\mathrm{NaCl}$ mixture was placed on the top of the compressed layer and the initial pressure was raised to 10 tons at $180^{\circ} \mathrm{C}$ for 10 minutes. The mould was cooled and the discs were taken out of the mould. Square section scaffolds, with $4.5 \mathrm{x}$ $4.5 \mathrm{~mm}$ cross-section and a reference height of $9 \mathrm{~mm}$, were cut from the obtained discs.

The leaching was performed on round bottomed flask with 20 times (by volume) of distilled water. The flasks were placed onto a mechanical shaker at $37^{\circ} \mathrm{C}$. The water was replaced every 4 hours. $\mathrm{NaCl}$ content was checked by aqueous silver nitrate solution. The leaching was continued until no precipitate by silver nitrate was detected.

\subsection{Characterisation of the scaffolds}

2.3.1. SEM analysis- A LEICA Cambridge S-360 (UK) scanning electron microscopy (SEM) analysis at $15 \mathrm{kV}$ was performed on scaffolds with all the components alone and the bi-layered scaffolds, before and after putting into simulated body fluid (SBF). Prior to analysis, each sample was gold coated.

2.3.2. Measurement of water uptake- Amount of water uptake of the scaffolds was investigated in by means of incubating the sliced scaffolds in distilled water at $37^{\circ} \mathrm{C}$. The samples were taken out at predetermined time intervals, blotted all the six sides of the cubes for 20 minutes and then weighted with an electronic balance. The wet specimens were dried in vacuum at $40{ }^{\circ} \mathrm{C}$ for 24 hours. The water uptake percentage was calculated as $\left(\mathrm{W}_{\mathrm{w}}-\mathrm{W}_{\mathrm{d}}\right) / \mathrm{W}_{\mathrm{d}} \mathrm{x} 100$, where $\mathrm{W}_{\mathrm{d}}$ was the mass of dried cube and $\mathrm{W}_{\mathrm{w}}$ was the mass of the wet cube after blotting for 20 minutes.

2.3.3. FTIR analysis- The FTIR spectra were recorded on a Perkin Elmer System 1600 FTIR with an attenuated total reflectance device from SPECAC (MKII Golden Gate, diamond crystal, penetration depth $20 \mu \mathrm{m}$, active area $0.8 \mathrm{~mm}^{2}$ ). Spectra were taken with a resolution of $2 \mathrm{~cm}^{-1}$ and were averaged over 24 scans. 
2.3.4. Bioactivity Test - The simulated body fluid (SBF) was prepared as the protocol described in the literature [11]. The SBF has been used for the in vitro assessment of bioactivity of synthetic materials by examining their apatite-forming ability. All the scaffolds were fish hooked and immersed in $50 \mathrm{ml} \mathrm{SBF}$ solution put in polypropylene tubes. These polypropylene tubes were placed in a water bath at $37^{\circ} \mathrm{C}$ for $1,3,7,14$ and 30 days. After removing the samples for the predetermined time periods, the specimens were thoroughly cleaned by distilled water and dried at $23{ }^{\circ} \mathrm{C}$ at $50 \%$ relative humidity.

\section{Results and discussion}

The method employed allowed to produce bi-layered constructs (Fig. 1) with independent and controllable porosity being essentially dependent on the amount and size of $\mathrm{NaCl}$ particles.

As expected, the porosity is higher on the cartilage-side (left) and the pore sizes are smaller. Moreover, the pores appear to be interconnected. On the other hand, the bone-side (right) exhibits larger pores but the interconnectivity is not quite evident. The densities of PLLA, PLLA/HA and PLLA/BG, SPLA50 were 0.38 $\pm 0.03,0.38 \pm .03,0.33 \pm 0.05$ and $0.48 \pm 0.03 \mathrm{~g} . \mathrm{cm}^{-3}$ respectively.

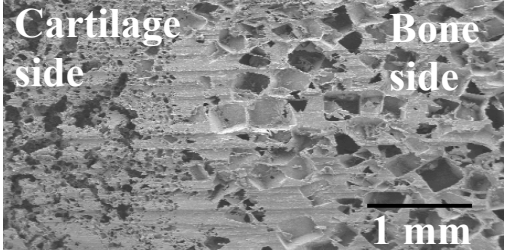

Fig. 1: Bi-layered construct

Water up-take capability porous scaffolds of PLLA, PLLA/HA, PPLA/BG and SPLA50, bi-layered

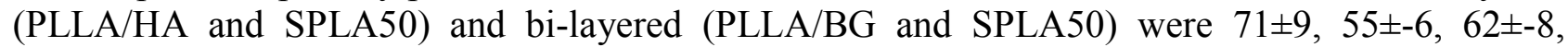
$250 \pm 25,150 \pm 6,145 \pm 7$ respectively. PLLA is a hydrophobic polymer; consequently the water uptake is low. As starch is strongly hydrophilic, the cartilage-side will absorb much more water. The water uptake after blotting was $250 \%$ in the SPLA50 layer resembling the natural hydration of cartilage. The bi-layered constructs had intermediate water uptake capabilities.
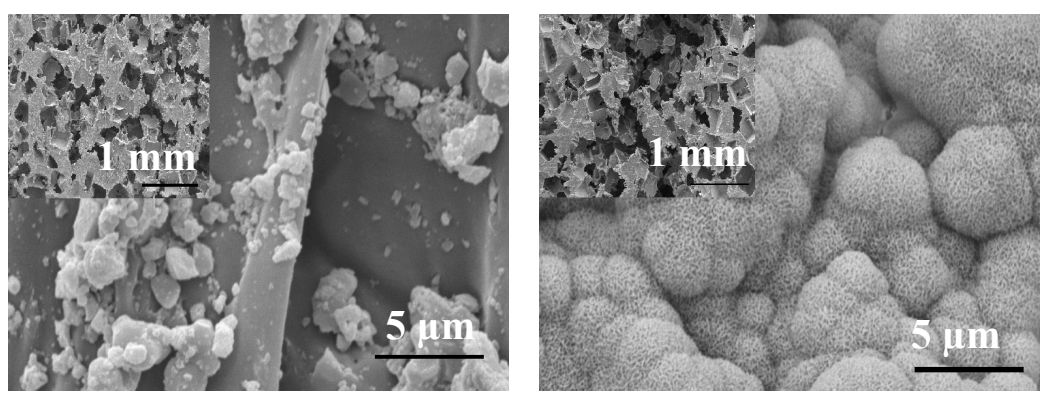

Fig. 2(a) and (b) - SEM images of PLLA/HA and PLLA/BG after incubation to SBF for 1 day. The inserts show the porous construct at lower magnification.

Both the porous construct of PLLA/HA and PLLA/BG showed no sign of apatite layer without incubation to SBF although $30 \mathrm{wt} \%$ of $\mathrm{HA}$ and $\mathrm{BG}$ were incorporated in the PLLA matrix. The apatite content over the surface of both constructs appeared to be increased from one to three days of incubation. However, the nature of apatite formation is dependent on the type of inorganic filler incorporated. PLLA/BG showed the complete coverage of the surface and a cauliflower like structure on day one. There is no sign of apatite formation on pure PLLA. SLA50 layer does not exhibit the formation of any Ca-P layer, being a positive result, as one should avoid any calcification in the cartilage region of the scaffold.

Fig. 3 shows the FTIR spectra of the developed mono-layered scaffolds and for pure HA.

For pure HA, the phosphate peak appeared near to $1000 \mathrm{~cm}^{-1}$. Pure PLLA did not show any sign of phosphate on surface in the incubation process. For PLLA/HA, there is a feeble sign of phosphate without incubation and phosphate is more prominent on day 3. These results are consistent with the SEM observations. Fig. 3(c) shows little sign of phosphate on day zero but strong presence were revealed on day one and day three. So, it is evident from the results that the presence of HA and especially of BG induces in-vitro bioactivity to porous PLLA constructs that is in accordance with previously reported data for PLLA/BG layer [12]. 


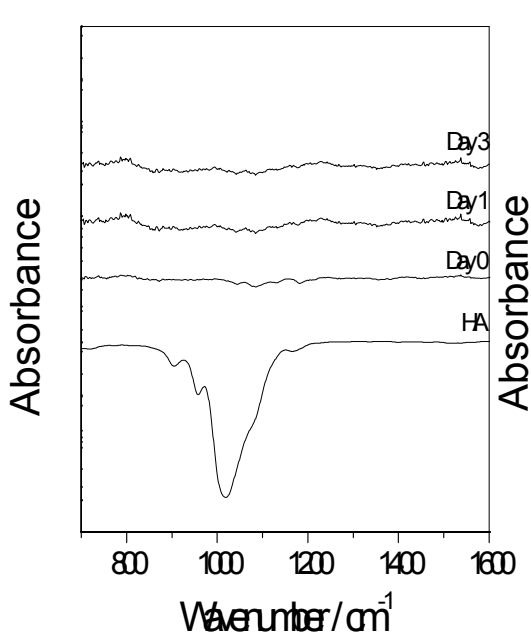

(a)

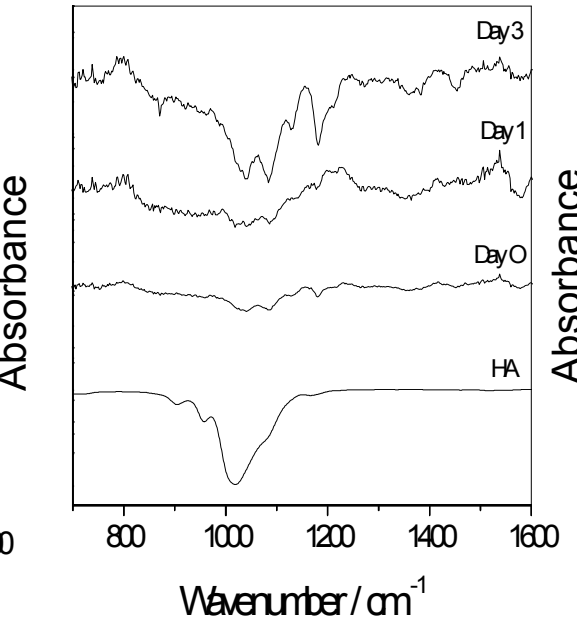

(b)

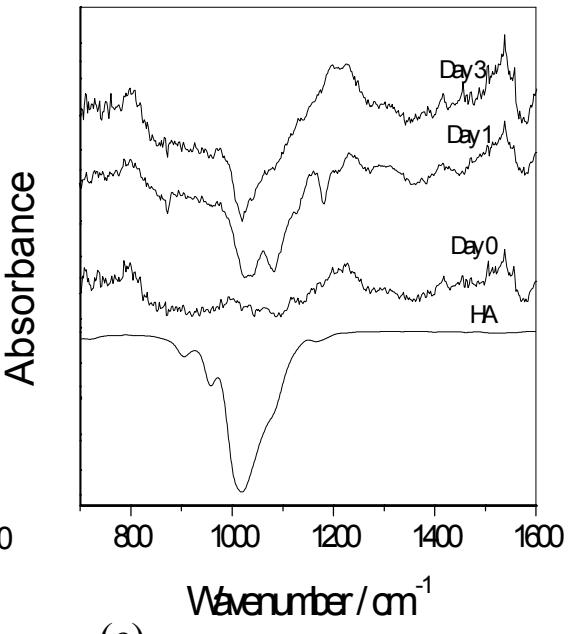

(c)

Fig. 3 - Representative FTIR spectra for (a) PLLA, (b) PLLA/HA and (c) PLLA/BG porous scaffolds after incubation to SBF for 0,1 and 3 days.

\section{Conclusions}

The developed constructs comprised two well integrated layers where the cartilage-like is composed by a blend of PLLA and starch, exhibiting swelling characteristics similar to human articular cartilage. Both composites, PLLA/HA and PLLA/BG exhibited a bioactive behaviour, being adequate for the bone-side.

Acknowledgements: Financial support for this work was provided by FCT, through the POCTI and FEDER programmes, and the project POCTI/FIS/61621/2004. S. Ghosh thanks FCT for awarding the $\mathrm{PhD}$ grant, SFRH/BD/12657/2003. This work was also partially supported by the European Union funded STREP Project HIPPOCRATES (NMP3-CT-2003-505758).

\section{References}

1. A.P. Newman: Am. J. Sports Med. Vol. 26 (1998), p. 309-24

2. D. Amiel, R.D. Coutts, M. Abel, W. Stewart, F. Harwood and W.H. Akeson: J Bone Joint Surg Am Vol. 67A (1985), p. 911-20

3. S.W. Odriscoll, F.W. Keeley and R.B. Salter: Bone Joint Surg Am Vol. 70A (1988), p. 595-606

4. S. Stevenson, G.A. Dannucci, N.A. Sharkey and R.R. Pool: Bone Joint Surg Am Vol. 71A (1989), p. 1297-1307

5. B. Obradovic, I. Martin, R.F. Padera, S. Treppo, L.E. Freed and G. Vunjak-Novakovic:

J.Orthopaed Res Vol. 19 (2001), p. 1089-1097

6. J.C. Middleton and A.J. Tipton: Biomaterials Vol. 21 (2000), p. 2335 - 46

7. S. Ishii, J. Tamura, T. Furukawa, T. Nakamura, Y. Matsusue, Y. Shikinami and M. Okuno: J. Biomed Mater Res Vol. 66B (2003), p. 539-47

8. K. Zhang, Y. Ma and L.F. Francis: J. Biomed Mater Res Vol. 61 (2002), p. 551-63

9. W.C. Puelacher, D. Mooney, R. Langer, J. Upton, J.P. Vacanti and C.A. Vacanti: Biomaterials Vol. 15 (1994), p. 774-78

10. A.P. Marques, O.P. Coutinho and R.L. Reis: J. Mater. Sci. - Mater Med (2005)

11. T. Kokubo, H. Kushitani, S. Saka, T. Kitsugi, T. Yamamuro: J. Biomed Mater Res Vol. 24

(1990), p. 721-34

12. B.S. Chang, C.K. Lee, K.S. Hong, H.J. Youn, H.S. Ryu, S.S. Chng and K.W. Park:

Biomaterials Vol. 21 (2000), p. 1291-98 